引理 1 任给一 $M \in \Lambda^{0}$, 存在唯一的 context $C\left[\right.$ ] 及 $F(W)$ 中的一组元素 $P_{1}, P_{2}$, $\cdots, P_{k}$ 使得 $C[$ ] 中无 $F(W)$ 中的项出现且 $M$ $\equiv C\left[P_{1}, P_{2}, \cdots, P_{k}\right]$.

引理 2 设 $G W \rightarrow \overrightarrow{\beta \eta} N, N$ 是 $\beta \eta$ 范 式. 则对任 $M$ 都有 $G M \rightarrow \vec{\beta} \rightarrow$

引理 3 对任 $X, Y, Z, U, V$ 都有

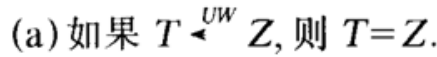

(b) 如果 $Z \stackrel{U W}{\rightarrow} F$, 则 $Z=F$.

(c) 如果 $X \stackrel{U V}{\longrightarrow} Y$, 则 $\exists Z(X \stackrel{U V}{\longleftarrow} Z \stackrel{U V}{\rightarrow} Y)$.

(d) 如果 $X \stackrel{U W}{\rightarrow} Z \stackrel{U W}{\stackrel{W}{*}} Y$, 则 $X \stackrel{U W}{\rightarrow} Y$.

引理 4 对任 $U \in \Lambda, \rightarrow(T \stackrel{U W}{\sim} F)$.

\section{参考文献}

1 Barendregt H P. The Lambda Calculus: Its Syntax and
Semantics. Ist ed. Amsterdam: North-Holland, 1984. $50 \sim 200$

2 Baeten J, Boerboom B. $\Omega$ can be anything it should not be. Indagationes Math, 1979, 41:111 120

3 Jacopini G. i-calculus and computer science thoery. Lecture Notes in Computer Science, 1975, 37:213 220

赵希顺

(河湳师范大学数学系, 新乡 453002)

$$
\text { 蒋 颖 王 驹 }
$$

(中国科学院软件研究所, 北京 100080)

*国家白然科学基金资助项目

1) 黄甘园, 蒋 顷等. U- 循坏项与易项 (待发表)

\title{
离子声波漂移不稳定性的实验研究 *
}

当电子温度 $T_{e}$ 远高于离子温度 $T_{i}$, 使电 子相对于离子漂移速度 $V_{d}$ 超过临界值 $V_{d c}$ 时, 电子分布函数在离子声波速度 $V_{\varphi}$ 处的导 数会大于零, 离子声波则变成不稳定的. 在 长波极限条件下, 可得到离子声波的增长率 $\gamma(K)$ 为

$$
\begin{aligned}
\gamma(K)= & -\left(\frac{\pi}{8}\right)^{1 / 2}\left(\frac{m}{M}\right)^{1 / 2} K\left[\left(V_{\varphi}-V_{d}\right)+V_{\varphi} \times\right. \\
& \left.\left(\frac{M}{m}\right)^{1 / 2}\left(\frac{T_{e}}{T_{i}}\right)^{3 / 2} \exp \left(-\frac{T_{e}}{2 T_{i}}\right)\right], \quad(1)
\end{aligned}
$$

式中 $m, M$ 分别为电子和离子质量, $K$ 为波 数. 令 $\gamma(K)=0$, 得到临界漂移速度 $V_{d c}$ 为

$$
\begin{aligned}
V_{d c}= & V_{\varphi}\left[1+\left(\frac{M}{m}\right)^{1 / 2}\left(\frac{T_{e}}{T_{i}}\right)^{3 / 2} \times\right. \\
& \left.\exp \left(-\frac{T_{e}}{2 T_{j}}\right)\right],
\end{aligned}
$$

即 $V_{d}<V_{d c}$ 时波是稳定的, 而 $V_{d}>V_{d c}$ 时则是 不稳定的, 这就是离子声波漂移不稳定性.
实验在双等离子体装置中进行 $(\varnothing 400 \times 1400 \mathrm{~mm})^{[1]}$, 氩气龙强 $P \approx$ $2.67 \times 10^{-2} \mathrm{~Pa}$, 等离子体浓度 $5 \times 10^{8} \mathrm{~cm}^{3}$, $T_{c} \sim 1 \mathrm{eV}, T_{e} / T_{i} \sim 15$, 电子和离子热速度分 别为 $V_{e} \sim 4.2 \times 10^{7} \mathrm{~cm} / \mathrm{s}, V_{i} \sim 4 \times 10^{4} \mathrm{~cm} / \mathrm{s}$. 电子碰撞频率 $v_{e} \sim 1 \times 10^{5} \mathrm{~s}^{-1}$. 平均自由程: $\left(\sim 10^{3} \mathrm{~mm}\right)$ 大于等离子体犬度, 则可看成无 碰撞等离子体.

图 1 为测得的振幅空间分布结果 $(150 \mathrm{kHz})$. 可见, 靠近激发栅极很小振幅迅 速增长, 当振幅足够大时, 甚至会出现周期 性起伏的非线性效应 ${ }^{\prime \prime}$. 出现不稳定性现象 的频率 $100 \sim 200 \mathrm{kHz}$, 相应波数 $K$ 为 $4.2 \sim$ $12.6 \mathrm{~cm}^{-1}$, 波长 $\lambda$ 为 $5 \sim 15 \mathrm{~mm}, V_{\varphi}$ 为 $1.3 \times$ $10^{5} \mathrm{~cm} / \mathrm{s}$. $\lambda$ 远小于等离子体尺度, 故器壁影 响可以忽略. 产生不稳定的两真空室空间 电位差 $\Delta V_{A B}$ 为 $(0.1 \sim 0.5 \mathrm{~V})$, 则 $V_{d}$ 为 $(0.42 \sim 2.1) \times 10^{7} \mathrm{~cm} / \mathrm{s}$.

设振幅指数增长, 由图 1 可得增长率 $\gamma$ $(K)$ 为 $6 \times 10^{4} \sim 1.5 \times 10^{5} \mathrm{~s}^{-1}$. 频率较低时 $\gamma$ 
较小, 在 $150 \sim 160 \mathrm{kHz}$ 达到极大值 $1.5 \times$ $\times 10^{5} \mathrm{~s}^{-1}$, 在频率较高时 $\gamma$ 有下降趋势. 实 验结果与理论比较可得出如下结论: (1) $V_{e} \gg V_{\varphi} \gg V_{i}$ 的结果说明, 电子和离子朗道 阻尼都较小. 将 $V_{\varphi}, T_{e} / T_{i}$ 等代人 (2) 式, 得 到临界电子漂移速度 $V_{d c}$ 为 $1.5 \times 10^{6} \mathrm{~cm} / \mathrm{s}$. 可 见实验测得的 $V_{d}$ 大于理论值 $V_{d c}$, 故我们的 实验条件在不稳定区域. (2) 将 $V_{\varphi}, V_{d}$ $T_{e} / T_{i}, K$ 代人 (1) 式, 算出增长系数 $\gamma(K)$ 为 $(1.9-5.8) \times 10^{5} \mathrm{~s}^{-1}$. 可见, 理论与实验结果在 量级上是一致的. 二者的差异归因于实验的 误差和理论的缺陷, 理论没有考虑各种可能 的耗散效应, 因此理论的 $\gamma(K)$ 随频率单调增 加. 而实验中低频段波长较长使碰撞的影响 相对较强, 造成低频范围测得之增长系数下 降. 随着频率增加, 使朗道阻尼影响增强, 则高频段增长系数也会下降. 这些因素的影 响, 使高频和低频段不仅不稳定现象难以出 现、甚至连普通的离子声波也难激发.

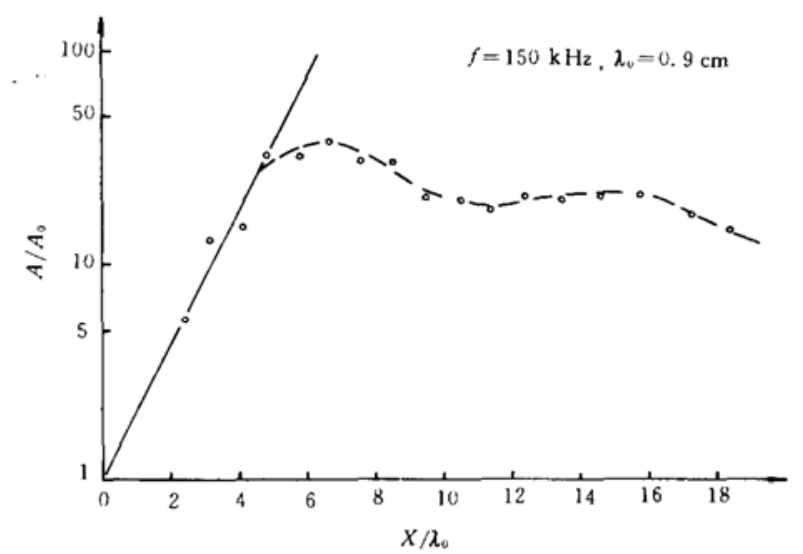

图 1 离子声波振幅随空间位置的分布 $X$ 为接收探针离激发棚极之距离; $A$ 为干涉仪输出, 其正比 于离子声波振幅; $A_{0}$ 为 $X=0$ 处之 $A$ 值

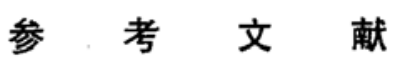

1 王文清. 离子声波非线性朗道阻尼的实验研究. 科 学通报, 1990, 35(11): 817

\section{王文清}

(北京大学地球物理学系, 北京 100871)

*国家自然科学基金资助项目

\section{人红细胞带 3 蛋白结构域间的通讯}

带 3 蛋白 (Band 3) 是人红细胞膜上的主 要蛋白质, 具有阴离子转运的功能. 它是目 前被研究得最广泛的膜蛋白之一. 从结构上 看, Band 3 可分成两个结构域. 其中膜结构 域负责其阴离子转运功能, 而细胞质结构域 则与细胞膜骨架蛋白 (如针蛋白 ankyrin 等) 相联从而起到稳定膜骨架的作用. 尽管 细胞质结构域对于 Band 3 膜结构域的阴离 子转运功能并不是必需的, 然而最近有些证 据表明这两个结构域之间可能存在一定的联 系, 但迄今为止人红细胞 Band 3 两个结构 域之间通讯的分子机制和结构基础仍不清 楚.

近年来我们在研究 Band 3 结构与功能
的调节时发现, $\mathrm{Zn}^{2+}(\mu \mathrm{mol} / \mathrm{L})$ 可抑制 Band 3 的阴离子转运活性; 而生理浓度范围 $(0.5 \sim$ $1 \times 10^{-6}$ ) 的 $\mathrm{Se}$ (硒) 则对 Band 3 具有激活作 用. 若用胰蛋白酶切去 Band 3 的细胞质结 构域则 $\mathrm{Zn}^{2+}$ 和 $\mathrm{Se}$ 的作用均消失. 原子吸收 光谱测定的结果表明 Band 3 细胞质结构域 存在着一个 $\mathrm{Zn}^{2+}$ 的结合位点. 而 N-3(- 芘)马来酰胺对 Band 3 荧光标记的结果则表明 细胞质结构域中部的 2 个半胱氨酸 (Cys201, 317) 可能是 Se 的结合位点. 蛋白质内源菼 光光谱和碘化钾对 Band 3 的内源苂光猝灭 的结果均表明 $\mathrm{Zn}^{2+}$ 或 Se 可诱导 Band 3 细 胞质结构域发生不同的构象变化. 而竹红菌 乙素 (一种从竹红菌中纯化的芘醌类衍生 